\title{
Incidence and risk factors of gestational diabetes mellitus in antenatal mothers in Goa, India
}

\author{
Rini R. Naik ${ }^{1 *}$, Guruprasad Pednekar ${ }^{1}$, Jagadish Cacodcar $^{2}$
}

\begin{abstract}
${ }^{1}$ Department of Obstetrics and Gynecology, ${ }^{2}$ Department of Preventive and Social Medicine, Goa Medical College, Goa, India
\end{abstract}

Received: 04 December 2018

Accepted: 29 December 2018

\author{
*Correspondence: \\ Dr. Rini R. Naik, \\ E-mail: rininaik123@gmail.com
}

Copyright: (c) the author(s), publisher and licensee Medip Academy. This is an open-access article distributed under the terms of the Creative Commons Attribution Non-Commercial License, which permits unrestricted non-commercial use, distribution, and reproduction in any medium, provided the original work is properly cited.

\begin{abstract}
Background: Gestational Diabetes Mellitus (GDM) accounts for almost $90 \%$ of all pregnancies complicated by diabetes. Its prevalence in the Indian population ranges from $5.5 \%$ to $11.5 \%$.

Methods: The present study was undertaken to assess the incidence of GDM among antenatal mothers admitted in Goa Medical College for a period of 18 months and also to study the risk factors associated with GDM amongst such cases.

Results: Present study revealed that among 7717 antenatal mothers who were admitted and delivered in our Hospital, 424 women were diagnosed with GDM. Of these women diagnosed with GDM, almost one third were in the age group of $31-35$ years $(35.8 \%) ; 56.2 \%$ of these women were multigravidas. Advancing maternal age, rising parity, obesity, family history of Diabetes Mellitus (DM) and past history of GDM were among the risk factors found to be significantly associated with GDM.

Conclusions: The incidence of GDM in present study population was 5.49\%. Advancing maternal age, maternal obesity, family history of DM and past history of GDM were found to be important determinants of GDM.
\end{abstract}

Keywords: Family history, Gestational diabetes mellitus, Obesity, Past history of GDM

\section{INTRODUCTION}

Gestational Diabetes Mellitus (GDM) is defined as any degree of carbohydrate intolerance which results in hyperglycaemia of variable severity with its onset or first recognition during pregnancy irrespective of whether the diabetes persists after pregnancy. ${ }^{1}$

According to the International Diabetes Federation (IDF), Diabetes Atlas 2017, the global prevalence of hyperglycaemia in pregnancy is $16.2 \%$ of which $86.4 \%$ of cases were due to GDM. South East Asia region had 6.9 million live births being affected by hyperglycaemia in pregnancy; with an estimated prevalence of $24.2 \%{ }^{2}$ Studies have shown that there has been a rising trend of
GDM in India, the prevalence ranging from 5.5\% during $2005-2006$ to $11.5 \%$ during $2013-2014 .^{3,4}$ This is probably associated with advancing maternal age, increased prevalence of maternal obesity, sedentary lifestyle and physical inactivity.

Goa being a former Portuguese colony is known to be the most westernized state in India and harbours multiple determinants responsible for development of GDM. However, no such large hospital based prospective studies have been published from Goa to assess the incidence of GDM and its risk factors. Hence the present study was taken up with the aim of determining the incidence of GDM among antenatal mothers admitted and delivered at Goa Medical College (GMC) and to 
study the correlation of GDM with various risk factors like maternal age, maternal obesity, multiparty, family history of DM, previous history of GDM, macrosomia and bad obstetric history.

\section{METHODS}

The present study was a large hospital based longitudinal study conducted in the Department of Obstetrics and Gynaecology, at GMC for a period of 18 months (November 2014-April 2016), upon the approval of the Institutional Ethics Committee (IEC) and an Informed consent obtained from the study participants.

\section{Inclusion criteria}

- The study participants included all the antenatal mothers who were registered in the antenatal clinic of GMC as well as those referred from other health care facilities for delivery at GMC during the above study period.

- The study participants who gave informed consent were then divided into 2 groups: Group A-antenatal women diagnosed with GDM and Group B-equal number of randomly chosen antenatal women without GDM who have delivered in the hospital during the same time period.

\section{Exclusion criteria}

- Antenatal mothers with overt diabetes mellitus, major chronic diseases like cardiac failure, hepatorenal failure and tuberculosis and with multiple gestation

- Antenatal mothers who were following up in GMC but delivered elsewhere.

The antenatal mothers who were included in the study were universally screened for GDM using oral glucose challenge test (OGCT) with 50g glucose at the first visit. $50 \mathrm{~g}$ of oral glucose load was given to all antenatal women regardless of time of the day or last meal. Plasma glucose level was measured at the end of 1 hour.

A value of more than $140 \mathrm{mg} / \mathrm{dl}$ was considered abnormal and followed up with Oral Glucose tolerance test (OGTT) with $100 \mathrm{~g}$ glucose. If the values were normal, then the screening test was repeated at 24-28 weeks of gestation. Antenatal women having any high-risk factors were directly subjected to OGTT with $100 \mathrm{~g}$ glucose at the first antenatal visit. They were called after overnight fasting of 8 hours. A fasting venous blood sample was drawn first to test for fasting blood sugar level. A $100 \mathrm{~g}$ oral glucose load was given usually prepared as a $50 \%$ glucose solution. Subsequent venous blood samples were drawn exactly at 1, 2 and 3 hours after the subject started to drink glucose. Hexokinase-mediated reaction Roche/Hitachi Modular P Chemistry Analyzer was used for processing of the blood samples. Carpenter and Coustan cut off values (Table 1) were used for diagnosis of GDM. ${ }^{5}$

Table 1: Carpenter and Coustan criteria.

\begin{tabular}{|l|l|}
\hline Fasting & Plasma glucose values $(\mathrm{mg} / \mathrm{dll})$ \\
\hline 1 hour & 95 \\
\hline 2 hours & 180 \\
\hline 3 hours & 155 \\
\hline $\begin{array}{l}\text { If any of the above } \\
\text { patient is labelled as GDM }\end{array}$ \\
\hline
\end{tabular}

A detailed history and a thorough clinical examination of the study participants were carried out. The study participants were screened for risk factors eg Age $>35$ years, obesity (Body mass index $>30 \mathrm{mg} / \mathrm{m}^{2}$ calculated at the first antenatal visit), family history of DM, history of GDM in the previous pregnancy, history of macrosomia in the previous pregnancy and Bad Obstetric History (BOH). $\mathrm{BOH}$ implied previous unfavorable fetal outcomes, such as 3 or more successive spontaneous abortions, early neonatal deaths, stillbirths, intrauterine growth restriction (IUGR) and congenital anomalies in the previous pregnancies. ${ }^{6}$ The results were analyzed using SPSS (version 22). Chi square test was applied as a test of significance and $p$ value of $<0.05$ was considered as statistically significant.

\section{RESULTS}

Among 7,717 antenatal mothers who were admitted and delivered at Goa Medical College during the study period, 424 women had GDM. The incidence of GDM in present study was thus $5.49 \%$.

Table 2: Comparison of Age distribution in GDM AND non- GDM groups.

\begin{tabular}{|l|l|l|l|l|}
\hline Age (years) & No. of antenatal women & Percentage & No. of antenatal women & Percentage \\
\hline$<20$ & 0 & 0 & 34 & 8.0 \\
\hline $20-25$ & 36 & 8.4 & 134 & 31.6 \\
\hline $26-30$ & 148 & 34.9 & 128 & 30.1 \\
\hline $31-35$ & 152 & 35.8 & 110 & 25.9 \\
\hline$>35$ & 88 & 20.7 & 18 & 4.2 \\
\hline
\end{tabular}


Majority of the women in the study were in the age group of $26-30$ years $(65 \%)$. Table 2 depicts that the proportion of GDM in the present study increased with advancing age $(\mathrm{p}=0.0000)$.

Among the women with GDM, 152 (35.8\%) women were in the age group of 31- 35 years. The mean age at detection of GDM was found to be 31.2 years.

184 patients in the GDM group were nulliparous, whereas in the non GDM group 176 patients were nulliparous.

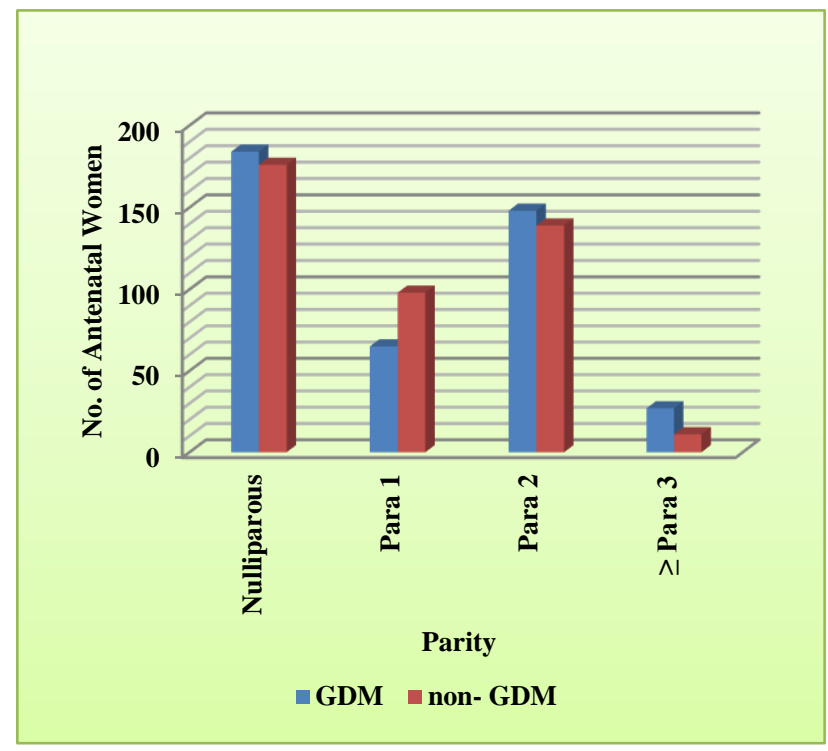

Figure 1: Comparison of parity in GDM and nonGDM groups.

Thus, the percentage of nulliparous patients in the study with GDM was $51 \%$.

Out of 38 patients who were $\geq$ Para 3 in the study the majority i.e. $27(71 \%)$ had GDM. The association between rising parity and GDM was statistically significant $(\mathrm{p}=0.0128)$.

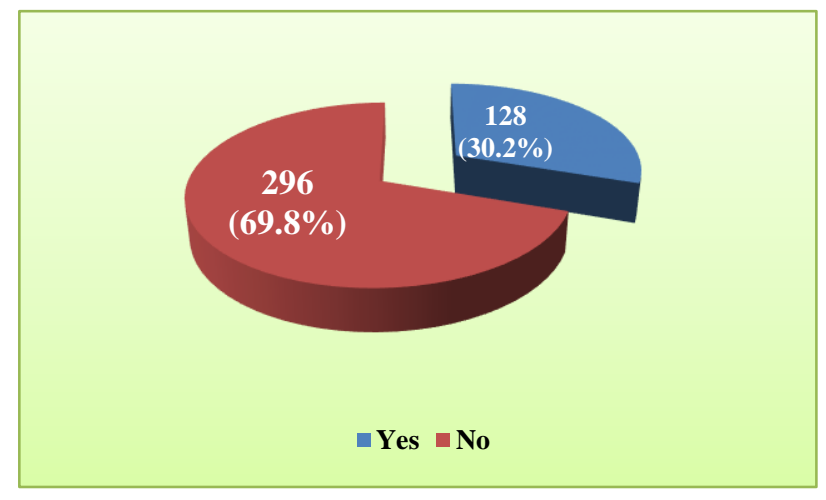

Figure 2: Percentage of obese mothers with GDM.

128 antenatal mothers $(30.2 \%)$ in the GDM group were obese. Among all the 168 obese antenatal mothers in the study, 128 (76\%) had developed GDM, thus indicating that maternal obesity was a significant risk factor for developing GDM $(p=0.0000)$. The present study showed that the prevalence of GDM was higher amongst women with family history of diabetes mellitus (DM). As seen in Table 3, majority of the antenatal mothers i.e. 256 women $(60.3 \%)$ with GDM had family history of DM among their first-degree relatives.

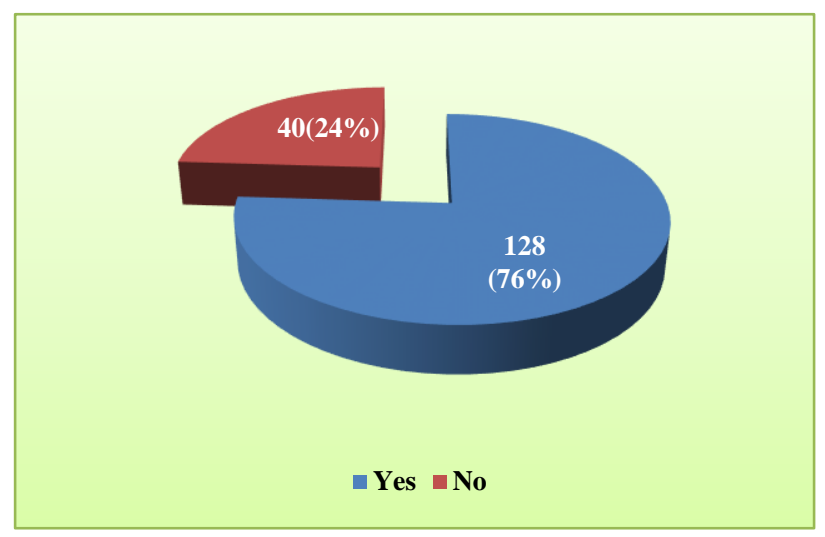

Figure 3: Percentage of mothers with GDM having obesity.

This finding was statistically significant $(p=0.0001)$. As seen in Table 4, risk factors associated with previous obstetric history such as past history of GDM, macrosomia in previous pregnancy and $\mathrm{BOH}$ were more prevalent in the GDM group as compared to non- GDM group.

Table 3: Family history of DM in GDM and nonGDM groups.

\begin{tabular}{|c|c|c|c|c|}
\hline $\begin{array}{l}\text { Family } \\
\text { history of } \\
\text { DM }\end{array}$ & $\begin{array}{l}\text { No. of } \\
\text { antenatal } \\
\text { mothers } \\
\text { with GDM }\end{array}$ & $\%$ & $\begin{array}{l}\text { No. of } \\
\text { antenatal } \\
\text { mothers } \\
\text { without GDM }\end{array}$ & $\%$ \\
\hline Yes & 256 & 60.3 & 198 & 46.6 \\
\hline No & 168 & 39.6 & 226 & 53.3 \\
\hline
\end{tabular}

However only past history of GDM was a statistically significant observation in the study population with GDM $(\mathrm{p}=0.0000)$.

Table 4: Past obstetric history in the study population.

\begin{tabular}{|c|c|c|c|}
\hline & $\begin{array}{l}\text { No. of } \\
\text { antenatal } \\
\text { mothers with } \\
\text { GDM }\end{array}$ & $\begin{array}{l}\text { No. of } \\
\text { antenatal } \\
\text { mothers } \\
\text { without GDM }\end{array}$ & $\begin{array}{l}\mathbf{P} \\
\text { value }\end{array}$ \\
\hline $\begin{array}{l}\text { Past history } \\
\text { of GDM }\end{array}$ & $101(23.8 \%)$ & $2(0.4 \%)$ & 0.0000 \\
\hline $\begin{array}{l}\text { History of } \\
\text { macrosomia } \\
\text { in previous } \\
\text { baby }\end{array}$ & $9(2.1 \%)$ & $4(0.9 \%)$ & 0.3027 \\
\hline $\mathrm{BOH}$ & $18(4.2 \%)$ & $10(2.3 \%)$ & 0.1785 \\
\hline
\end{tabular}




\section{DISCUSSION}

Gestational Diabetes Mellitus is a transient disorder in pregnancy and usually resolves after delivery. However, women with GDM are potential candidates for developing Type 2 diabetes mellitus in future; and so are their children. ${ }^{2,7}$ Women diagnosed with GDM are at increased risk of adverse pregnancy outcomes. Thus, universal screening of GDM, its early detection and appropriate management enables us to improve maternal and neonatal outcomes.

The incidence of GDM is increasing globally. IDF in 2017 has reported that a total of 21.3 million live births were affected by hyperglycemia in pregnancy. ${ }^{2}$ India dubbed as "Diabetes capital of the world" has highest incidence of GDM, with the relative risk of Indian women developing GDM being 11.3 times more in comparison with Caucasian women. ${ }^{8}$ In the present study the incidence of GDM was found to be $5.49 \%$ which was comparable to other studies conducted in India by Varija Thathagari et al in Mysore (5.5\%), Maxima Anand et al in Punjab (5.3\%) and Kalpana Varma in Uttar Pradesh $(6.72 \%) .^{3,9,10}$ However, studies done by Shridevi AS et al in Davangere $(11.5 \%$ ), Karnataka and Pallav Parikh et al in Gujarat (13.79\%) showed higher incidence of GDM. ${ }^{4,11}$ This shows that there are wide variations in the incidence of GDM as reported in different parts of India. This variation could be due to technical fallacies likes sample being drawn from urban or rural population, ethnicity, socioeconomic disparities and different diagnostic criteria used. Shridevi et al and P. Parikh et al used DIPSI guidelines for diagnosis of GDM while the other studies comparable to present study used OGTT with $100 \mathrm{~g}$ glucose. ${ }^{4,11}$

In the present study, the incidence of GDM was found to be significantly rising with advancing maternal age, with highest incidence of GDM in the age group of 31-35 years $(35.8 \%)$. Varija Thathagari et al, Shridevi AS et al, Maxima Anand et al and Kalpana Varma et al also reported that advancing maternal age is a significant risk factor for developing GDM..$^{3,4,9,10}$ However Pallav Parikh et al in Gujarat did not observe such a significant finding. ${ }^{11}$ This may be due to disparity in the study design with limitation to rural population in Gujarat. The present study demonstrated that $43.4 \%$ of patients were nulliparous which was similar to the study conducted Varija Thathagari et al in Mysore $(40.5 \%)$ and $56.6 \%$ of GDM patients were multiparous which was comparable to study conducted by Varija Thathagari et al $(59.5 \%) .^{3}$ In both the studies, it was seen that a higher percentage of primigravidas had GDM as compared to other studies because women in India in recent times, especially in urban areas got married late and hence conceived at a relatively older age. Thus, a high percentage of primigravidas are developing GDM in India due to advancing maternal age at conception. Amongst the 38 women who were > para 3 in present study, $71 \%$ had GDM thus making rising parity a significant risk factor for GDM. However, Varija Thathagari et al, Kalpana Varma et al and Pallav Parikh et al did not find such association. ${ }^{3,10,11}$

Obesity has become a major public health problem in recent times in India, which is still battling malnutrition, due to changing lifestyles, physical inactivity and westernized diets and culture especially in the urban areas of India. In present study it was observed that $30.2 \%$ of antenatal mothers with GDM were obese, thus making maternal obesity a significant risk factor for the development of GDM. Increased prevalence of GDM in women with higher BMI was found to be a significant finding in all the above-mentioned studies., , $^{3,9-11}$ Present study showed that majority of the antenatal mothers with GDM $(60.3 \%)$ had family history of diabetes mellitus in their first-degree relatives. Studies conducted in other parts of India have documented similar observation thus making family history of DM an important variable in the etiology of GDM. . $^{3,4,9,10}$

The present study revealed that risk factors associated with obstetric history such as past history of GDM $(23.8 \%)$, history of macrosomia in previous pregnancy (2.1\%) and Bad Obstetric History (4.2\%) were more common in GDM than non-GDM group; however, amongst all these variables only past history of GDM was found to be significantly associated with GDM. Varija Thathagari et al, Shridevi AS et al and Kalpana Varma et al found all these three risk factors associated with previous pregnancy to be statistically significant determinants for developing GDM..$^{3,4,10}$ On the other hand, Maxima Anand et al found that past history of GDM and macrosomia were not statistically significant risk factors (probably due to smaller size of study population), but $\mathrm{BOH}$ was found to be significantly associated with GDM. ${ }^{9}$ This variation may be due to the fact that many other determinants influence the incidence of $\mathrm{BOH}$ like hypertension, hypothyroidism, overt diabetes mellitus, cervical incompetence, etc. The dissimilarity in the different study settings may be responsible for these variations in these study results.

\section{CONCLUSION}

The incidence of GDM in present study was $5.49 \%$. The present study highlights that the important determinants for developing GDM are advancing maternal age, rising parity, maternal obesity, family history of DM and past history of GDM. A meticulous history must be obtained from all antenatal mothers. Those identified with high risk factors should be directly subjected to diagnostic tests for GDM. Amongst all the risk factors for GDM, obesity is the only factor which is modifiable. Hence pregnant mothers should be counselled regarding lifestyle modifications in order to prevent obesity and GDM.

Funding: No funding sources Conflict of interest: None declared

Ethical approval: Not required 


\section{REFERENCES}

1. American Diabetes Association, Classification and diagnosis of diabetes, Diabetes Care.2015;38(1):S8S16.

2. International Diabetes Federation. IDF Diabetes Atlas, 8th edition. Brussels, Belgium: International Diabetes Federation, 2017. Available at http://www.diabetesatlas.org

3. Thathagari V, Doddaiah V, Raghavenda B. A study of prevalence and determinants of gestational diabetes mellitus. Int J Reprod Contracept Obstet and Gynaecol. 2016;5(5):1331-5

4. Shridevi AS, Prabhudev P, Madhusoodana R. Bhovi, A clinical study of prevalence of gestational diabetes mellitus and associated risk factors at a tertiary care centre in Karnataka, India. Int J Reprod Contracept Obstet Gynaecol. 2015;4(6):1840-5.

5. Carpenter MW, Coustan DR, and Criteria for screening tests for gestational diabetes. Am J Obstet Gynecol. 1982,144(7):768-73.

6. Champa Koppad, Lakshmi K.S. Immunological Causes of Bad Obstetric History. J Evidence Med Healthcare.2014;1(16):2086-99.
7. Dornhorst A, Rossi M. Risk and Prevention of Type 2 Diabetes in women with Gestational Diabetes. Diabetes Care. 1998;21(2):B43-9.

8. Seshiah V, Balaji V, Balaji MS, Sekar A, Sanjeevi $\mathrm{CB}$, Green A. One step procedure for screening and diagnosis of gestational diabetes mellitus. Diabetes. 2005;126:200.

9. Anand M, Anand M, Mahajan DS. To study the incidence of gestational diabetes mellitus and risk factors associated with GDM. International Journal of Advance Medicine. 2017;4(1):112-6.

10. Surwade VM, Sinha V, Kachhawa P. Prevalence of gestational diabetes and risk factors among women visiting antenatal clinic of A tertiary health care hospital. IOSR-JDMS. 2017;16(9):1-4.

11. Pallav Parikh, Asha Sha, Dinesh Joshi, Rushit Shah. Prevalence of gestational diabetes mellitus at tertiary care center. Int J Sci Res Pub. 2014;4(9):1-3.

Cite this article as: Naik RR, Pednekar G, Cacodcar J. Incidence and risk factors of gestational diabetes mellitus in antenatal mothers in Goa, India. Int $\mathbf{J}$ Reprod Contracept Obstet Gynecol 2019;8:586-90. 